\title{
NOUVELle
}

\section{Initiation de l'endocytose par vésicules de clathrine}

Des « sculpteurs

de membrane $\gg$ au travail

Emmanuel Boucrot, Harvey T. McMahon

Laboratoire de biologie moléculaire du MRC, Hills Road, CB2 OQH Cambridge, Royaume-Uni. eboucrot@mrc-Imb.cam.ac.uk
> L'endocytose par vésicules recouvertes de clathrine est une voie majeure d'internalisation dans les cellules de nombreux récepteurs transmembranaires ayant des rôles-clés dans de multiples fonctions cellulaires $[1,2]$. Cette voie d'endocytose contrôle l'entrée sélective de nombreux facteurs de croissance (hormones) et nutriments [2]. Elle est aussi détournée par certains virus et bactéries qui infectent les cellules de cette façon.

La formation de vésicules recouvertes de clathrine requiert l'action concertée de plusieurs dizaines de protéines. Les étapes nécessaires sont le tri et la concentration des récepteurs, la déformation de la membrane plasmique et le recrutement du manteau de clathrine pour former un puits recouvert de clathrine, et enfin la scission de la vésicule de la surface cellulaire (Figure 1). Les principales protéines impliquées lors de ces étapes sont l'adaptateur AP2, les triskèles de clathrine (voir plus loin et [33]), la mécano-enzyme dynamine et de nombreuses protéines dites accessoires ayant des fonctions diverses, comme l'aide au tri sélectif des récepteurs ou la déformation de la membrane plasmique $[3,4]$.

La clathrine est présente dans le cytosol sous forme de triskèles ${ }^{1}$ qui se polymérisent en cages formées d'hexagones et

${ }^{1}$ La clathrine est constituée de trois chaînes lourdes associées par leur domaine carboxy-terminal, chacune d'elles étant associée à une chaîne légère. Cet assemblage a été visualisé en microscopie électronique et appelé «triskèle » par ressemblance avec le symbole celte du mouvement perpétuel (repris de [33]). de pentagones (de forme similaire à un ballon de football) $[5,6]$. La clathrine ne peut pas se lier directement à la membrane plasmique ou aux récepteurs transmembranaires et sa polymérisation est dépendante de protéines adaptatrices, comme AP2 (Figure 1) [3]. Formée de quatre sous-unités $(\alpha 2, \beta 2, \mu 2$ et $\sigma 2)$, AP2 fait la connexion entre de nombreux récepteurs, les triskèles de clathrine et de nombreuses protéines accessoires $[3,7]$. Plusieurs dizaines d'autres protéines se lient soit à la clathrine soit à l'adaptateur AP2 et sont impliquées dans la formation des vésicules de clathrine [3, 4]. La fonction précise de nombre d'entre elles est toujours inconnue. Le détachement des vésicules de clathrine de la membrane plasmique requiert l'action de la dynamine, une mécano-enzyme qui induit la fission de la base (« au cou ») de la vésicule [8]. Enfin, le désassemblage du manteau de clathrine de la vésicule nouvellement formée est assuré par l'ATPase Hsc70, recrutée par la protéine auxiline (Figure 1) [9-11]. La vésicule poursuit alors son chemin vers les endosomes précoces, et les composants de la machinerie du manteau de clathrine sont de nouveau disponibles pour un cycle.

Les récents progrès technologiques, comme la vidéomicroscopie, la spectrométrie de masse et la déplétion ciblée de protéines par des ARN interférents ont permis d'acquérir une meilleure connaissance du processus de formation des vésicules de clathrine. L'utilisation de la vidéomicroscopie a permis l'observation en temps réel de la formation de vési- cules de clathrine individuelles [12-14]. Des études utilisant la spectrométrie de masse ont identifié de nombreuses nouvelles protéines présentes dans les vésicules de clathrine $[15,16]$ dont les fonctions ont été identifiées grâce à l'effet d'ARN interférents $[17,18]$. Cependant, plusieurs étapes de la formation des vésicules de clathrine restent incomprises, dont l'initiation, ou nucléation, des puits de clathrine.

Induction des vésicules de clathrine : le rôle controversé d'AP2

L'initiation de la formation de vésicules de clathrine est une étape difficile à étudier: les puits recouverts de clathrine se forment et deviennent des vésicules relativement rapidement (20 à 90 secondes), sont trop petits (environ $100 \mathrm{~nm}$ ) pour être observés directement par microscopie optique et se forment aléatoirement sur la surface des cellules $[19,20]$. L'adaptateur AP2 a longtemps été considéré comme responsable de la nucléation des puits de clathrine. $\varepsilon n$ effet, cet adaptateur tétramérique est idéalement placé au centre du réseau d'interactions protéiques impliquées dans la formation des vésicules de clathrine [3]. AP2 se lie simultanément aux motifs endocytiques des récepteurs transmembranaires via ses sous-unités $\mu 2$ and $\sigma 2$, et aux triskèles de clathrine via $\beta 2$ [4]. AP2 se lie aussi au phosphatidylinositol bisphosphate $\left(\mathrm{Pi}[4,5] \mathrm{P}_{2}\right)$, un lipide spécifique de la membrane plasmique, via $\mu 2$ et $\alpha 2$ et à de nombreuses protéines accessoires via $\alpha 2$ et $\beta 2$ [4]. Le rôle central d'AP2 a été 


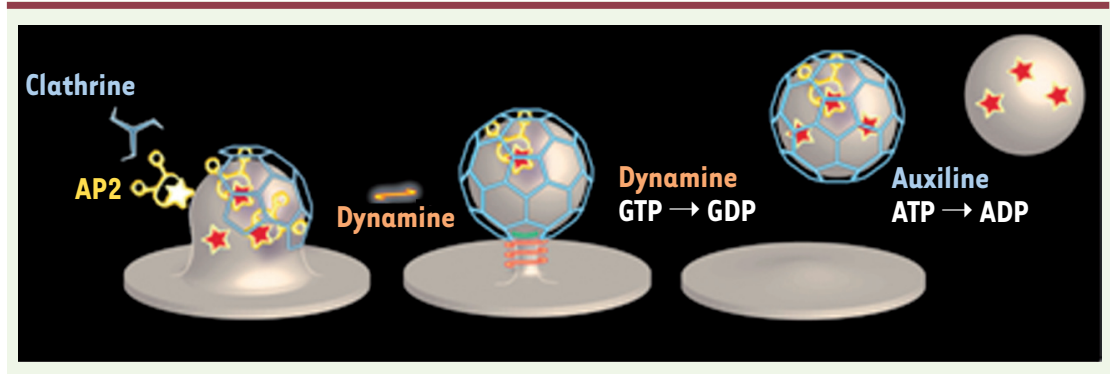

Figure 1. Modèle classique de la formation des vésicules de clathrine. L'adaptateur AP2 (jaune) se lie à la membrane plasmique en reconnaissant le lipide $\mathrm{Pi}(4,5) \mathrm{P}_{2}$ et des motifs endocytiques de certains récepteurs transmembranaires (étoiles rouges). Les triskèles de clathrine (bleu) ne peuvent pas lier directement la membrane plasmique et ne sont recrutés que par l'intermédiaire d'AP2 ou d'autres adaptateurs. La polymérisation du manteau de clathrine induit la déformation de la membrane plasmique et la formation d'un puits recouvert de clathrine. La mécano-enzyme dynamine se polymérise au cou de la vésicule naissante et, par hydrolyse de GTP, induit la scission de la vésicule. À ce stade, l'auxiline recrute Hsc70 qui induit la dépolymérisation du manteau de clathrine. La vésicule continue alors son chemin en route pour les endosomes précoces, et les composants de la machinerie de la clathrine sont libérés pour un nouveau cycle.

remis en question par les résultats d'une étude qui suggérait que la déplétion d'AP2 ne bloquait pas totalement la formation des vésicules de clathrine [21]. On pouvait donc prédire que d'autres protéines accessoires pourraient remplir le rôle d'AP2, tout du moins en son absence. Cependant, faute de preuves de la formation de vésicules de clathrine dépourvues d'AP2, et compte tenu de certains détails importants des protocoles expérimentaux employés, le rôle central et indispensable d'AP2 a été réétabli [3, 17, 22]. Néanmoins, même s'il est central, AP2 n'a pas une grande affinité pour la membrane plasmique $\left(\mathrm{Pi}[4,5] \mathrm{P}_{2}\right)$ et les récepteurs transmembranaires, caractéristiques attendues pour induire la formation de puits de clathrine. De plus, l'arrimage à la membrane des triskèles de clathrine par le biais des adaptateurs (AP2 ou autres) et leur polymérisation ne sont pas suffisants pour induire la courbure de la membrane plasmique nécessaire à la formation de puits, puis de vésicules. Ceci est bien illustré par les fréquents treillis plats de clathrine observés à la surface de nombreuses cellules [23]. Le rôle actif de certaines protéines capables de «sculpter» la membrane plasmique a été établi [24-27]. En revanche, parmi les protéines connues ayant ces propriétés, la plupart ont un rôle tardif lors de la transition, au moment où les puits de clathrine deviennent des vésicules.

\section{Les protéines $\mathrm{FCHo}$} sont des nucléateurs de la formation des vésicules de clathrine Parmi les protéines récemment associées à la formation des vésicules de clathrine, une nouvelle petite famille, celle des protéines FCHo (Fer/Cip4 homology domain-only), a montré des propriétés particulières. Dans des cellules épithéliales et neuronales mammifères, les protéines FCHo ont été détectées, par vidéomicroscopie et microscopie électronique, à la membrane plasmique avant même AP2 et la clathrine (Figure 2) [28]. Elles définissent ainsi l'endroit où les puits de clathrine se formeront. Ce sont les premières protéines à avoir été détectées, et elles précéderaient la mise en place de la machinerie de formation des vésicules de clathrine [28]. Chez l'homme, il existe trois protéines $\mathrm{FCHo}$, produits des gènes $\mathrm{FCHOl}$ (deux isoformes) et FCHO2. Chez la levure Saccharomyces cerevisiae, il existe un seul homologue, le gène SYPI (suppressor of yeast profilin deletion). La protéine Sypl a aussi été détectée au tout début de la formation des vésicules de clathrine [29, 30]. Contrairement aux autres protéines associées à la formation des puits de clathrine, les FCHo ne sont pas présentes dans les vésicules de clathrine, le «produit final » $[15,16]$. Elles ne sont plus détectées après la constriction du cou de la vésicule, juste avant son détachement [28]. Les manipulations des niveaux d'expression des protéines FCHo ont révélé l'influence directe de ces protéines sur la nucléation des vésicules de clathrine. Lorsque les niveaux des protéines FCHo sont diminués par ARN interférence, le nombre de puits de clathrine à la membrane plasmique chute de manière drastique [28]. II y a une inhibition parallèle de l'internalisation de ligands qui dépendent de la clathrine pour leur entrée dans les cellules, comme la transferrine (qui transporte le fer dans les cellules), le cholestérol (LDL) et le facteur de croissance EGF (epidermal growth factor). À l'inverse, la titration des niveaux d'expression des protéines FCHo par surexpression induit une augmentation parallèle du nombre de puits et vésicules de clathrine [28]. Comme il existe une corrélation directe entre la dose de protéines FCHo surexprimées et le nombre de vésicules de clathrine produites, cela suggère un rôle direct des protéines FCHo. Les vésicules de clathrine additionnelles sont productives car il y a une augmentation parallèle de l'internalisation de la transferrine dans les cellules. Les protéines $\mathrm{FCH}$ ont un rôle redondant car la déplétion simultanée de $\mathrm{FCHol}$ et $\mathrm{FCHo} 2$ est nécessaire pour inhiber la formation des vésicules de clathrine. À l'inverse, la surexpression de chacune des protéines est suffisante pour induire la formation de vésicules de clathrine additionnelles [28]. Ces études ont permis d'ajouter une étape 


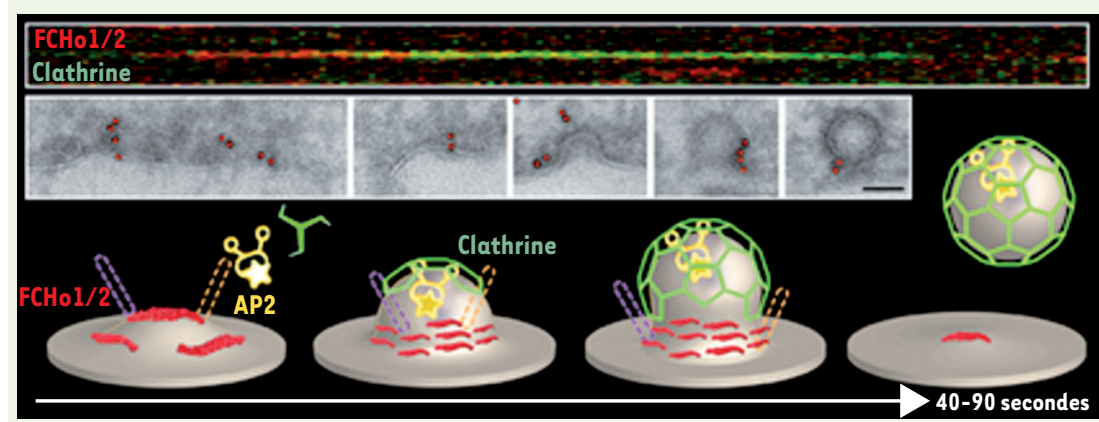

Figure 2. Les protéines FCHo initient la formation des vésicules de clathrine. En haut: en vidéomicroscopie (kymographe), les protéines FCHol et 2 (rouge) sont détectées avant la clathrine (vert). Au milieu: I'immunomarquage de $\mathrm{FCH}_{0} 2$ (points rouges) observé par microscopie électronique cryogénique (pour préserver les membranes) montre que $\mathrm{FCHo} 2$ est présent sur des surfaces planes, avant le recrutement d'un manteau de clathrine, puis se concentre au cou de la vésicule naissante.

En bas : nouveau modèle de formation des vésicules de clathrine. Les protéines $\mathrm{FCHol}$ et 2 (rouge) lient la membrane plasmique riche en $\mathrm{Pi}(4,5) \mathrm{P}_{2}$, recrutent les protéines Eps 15, Eps 15R (violet) et les intersectines (jaune), qui à leur tour regroupent et maintiennent les protéines FCHo et recrutent l'adaptateur AP2 et les triskèles de clathrine (vert), induisant l'initiation de la formation d'un puits de clathrine. Les protéines FCHo restent au cou de la vésicule naissante où leur activité de «sculpteur » de membrane est nécessaire pour la génération de la courbure requise pour la déformation de la membrane. Le reste du processus est le même que celui décrit en Figure 1. D’après [28].

supplémentaire en amont du modèle classique de formation des puits de clathrine (Figure 2).

\section{Comment les protéines $\mathrm{FCHo}$ induisent-elles la formation des vésicules de clathrine?}

Les trois protéines $\mathrm{FCH}$ o sont composées de domaines similaires : elles possèdent un domaine F-BAR (Fes/CIP4 homology$B A R)$ à leur extrémité amino-terminale et un domaine d'homologie à la sousunité $\mu$ de AP2, appelé $\mu$ HD ( $\mu$ homology domain) à leur extrémité carboxyterminale (Figure 3). Ce dernier est un domaine de séquence et de structure similaires à la sous-unité $\mu 2$ de l'adaptateur AP2, présent dans les protéines FCHo et SGIPI (SH3-containing GRB2-like protein 3-interacting protein 1) [29]. Les protéines $\mathrm{FCHo}$ ne se lient directement $\mathrm{ni}$ à AP2 ni à la clathrine [28]. En revanche, via leur domaine $\mu \mathrm{HD}$, elles forment un complexe direct avec plusieurs autres protéines impliquées dans la formation des vésicules de clathrine, en particulier Eps15 (epidermal growth factor receptor substrate 15) et Eps15R et les intersectines $[28,29]$. Ces dernières se lient aussi à AP2, à la clathrine et à plusieurs autres protéines importantes pour la formation de vésicules, telle la dynamine. Ces protéines sont recrutées aux zones qui deviendront des puits de clathrine aussi précocement que les protéines FCHo, et indépendamment d'AP2 [28]. La fonction et la concentration en zones définies des protéines $\mathrm{FCHo}$ dépendent d'Eps15, Eps15R et des intersectines, car la diminution simultanée de leur expression induit la même inhibition de la formation de puits de clathrine que la déplétion des protéines $\mathrm{FCHo}$. En revanche, les $\mathrm{FCH}$ o agissent en amont de ces protéines car, en absence des $\mathrm{FCH}$, elles ne sont pas recrutées à la membrane plasmique [28]. Les $\mathrm{FCHo}$ se lient à la membrane plasmique indépendamment de toute autre protéine grâce à leurs domaines F-BAR. Les protéines à domaines F-BAR forment une sous-classe de la famille des protéines à domaines $B A R$ (Bin/Amphiphysin/Rvs) [31]. Les domaines BAR sont des modules de dimérisation intermoléculaires en forme de croissant, ayant des faces concaves riches en résidus chargés positivement et pouvant lier les lipides des membranes cellulaires chargés négativement (Figure 3) [26]. $\varepsilon n$ plus de leur capacité à se lier aux membranes, les domaines BAR ont aussi celle de sentir et d'induire une courbure chez celles-ci [26]. Les domaines F-BAR peuvent également se lier aux membranes et induire une courbure, mais, en raison de la formation d'un angle moins prononcé, ils n'induisent pas de courbure aussi extrême que celle qu'in- duisent les domaines BAR [27, 32]. Le domaine F-BAR des protéines $F C H$ a une préférence pour le $\mathrm{Pi}(4,5) \mathrm{P}_{2}$ par rapport aux autres lipides [28], ce qui explique pourquoi ces protéines sont exclusivement recrutées à la membrane plasmique, qui est très riche en $\operatorname{Pi}(4,5) \mathrm{P}_{2}$. Lors d'expériences de tubulation in vitro, le domaine F-BAR de $\mathrm{FCHo} 2$ est capable de déformer des liposomes en tubes de diamètres allant de 130 à $18 \mathrm{~nm}$ selon la concentration de protéines [28]. Plusieurs autres protéines telles que l'amphiphysine, SNX9, FBP17, l'endophiline et l'epsine sont impliquées dans la formation de vésicules de clathrine et ont la capacité d'induire une courbure de la membrane $[25-27,31]$. En revanche, toutes ces protéines ont un rôle plus tardif que les $\mathrm{FCHo}$ et n'induisent pas la nucléation des puits de clathrine. Le domaine F-BAR des protéines $\mathrm{FCH}$ o joue un rôle crucial dans leurs fonctions. Des mutations visant à réduire leur dimérisation ou leur capacité à se lier aux membranes suffisent à abolir la capacité des protéines $\mathrm{FCHo}$ à induire la formation de puits de clathrine [28, 29]. De plus, la capacité des FCHo à «sculpter »la membrane plasmique à travers leurs domaines F-BAR est cruciale pour la progression des puits de clathrine en vésicules. En effet, deux mutations indépendantes qui abolissent 


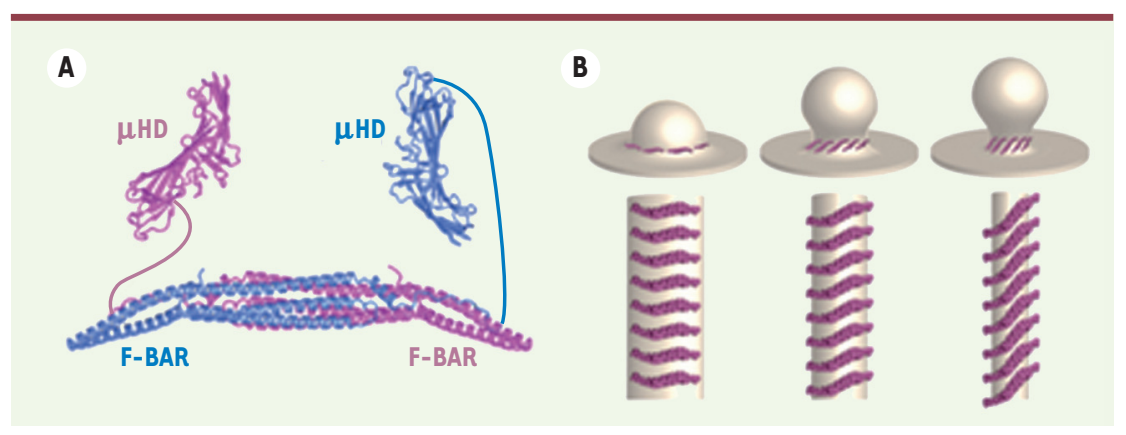

Figure 3. Les protéines $\mathrm{FCH}$ o sont des «sculpteurs de membrane ». A. Les protéines FCHo sont composées d'un domaine F-BAR (Fes/CIP4 homology$B A R)$ à leurs extrémités amino-terminales et un domaine d'homologie à la sous-unité $\mu$ de AP2, «HDD ( $\mu$ homology domain) à leur extrémité carboxy-terminale. Deux molécules de $\mathrm{FCHo} 2$ sont représentées ici (une bleue et une magenta), formant un dimère fonctionnel. La dimérisation des domaines F-BAR crée un domaine en forme de croissant, avec une face concave riche en résidus chargés positivement qui se lie et déforme les membranes. Les domaines $\mu H D$ se lient aux protéines Eps 15, Eps 15R et intersectines qui, à leur tour, recrutent l'adaptateur AP2 et les triskèles de clathrine. B. Les domaines F-BAR des protéines FCHo peuvent induire plusieurs degrés de courbure de la membrane plasmique. II existe un parallèle entre l'arrangement des domaines F-BAR de FCHo2 sur les tubes de diamètres allant de 130 à 18 nm qu'ils induisent in vitro (bas) et l'arrangement qu'ils pourraient avoir au cou de la vésicule naissante (haut). Les protéines FCHo restent au cou de la vésicule (voir images de microscopie électronique de la Figure 2) (d'après [28]).

la formation en tube des membranes par le domaine F-BAR (mais pas sa capacité à se lier aux membranes) bloquent la progression des puits de clathrine à un stade précoce [28].

Les protéines FCHo sont des nucléateurs des puits de clathrine à la membrane plasmique et induisent la courbure initiale nécessaire à la formation des puits. Cependant, de nombreuses questions restent encore sans réponse. y $a-t$-il d'autres protéines capables d'induire la formation des puits de clathrine? Comment les récepteurs sont-ils recrutés et concentrés dans les puits? Comment la formation des puits de clathrine estelle régulée? Les études des prochaines années vont vraisemblablement y répondre. $\diamond$

Nucleation of clathrin-coated pits: « membrane sculptors » at work

\section{CONFLIT D'INTÉRÊTS}

Les auteurs déclarent n'avoir aucun conflit d'intérêts concernant les données publiées dans cet article.

\section{REMERCIEMENTS}

Les auteurs remercient le Medical Research Council (MRC) et la Human Frontier Science Program Organization (HFSP) pour leur soutien et Mlle Wendy Tsang Man Pun pour la relecture de cette nouvelle.

\section{RÉFÉRENCES}

1. Doherty GJ, McMahon HT. Mechanisms of endocytosis. Annu Rev Biochem 2009 ; 78 : 857-902.
2. Scita G, Di Fiore PP. The endocytic matrix. Nature $2010 ; 463: 464-73$.

3. Schmid EM, McMahon HT. Integrating molecular and network biology to decode endocytosis. Nature 2007 ; 448: 883-8.

4. Traub LM. Tickets to ride: selecting cargo for clathrinregulated internalization. Nat Rev Mol Cell Biol 2009; $10: 583-96$.

5. Crowther RA, Pearse BM. Assembly and packing of clathrin into coats. J Cell Biol $1981 ; 91: 790-7$.

6. Fotin A, Cheng Y, Sliz P, et al. Molecular model for a complete clathrin lattice from electron cryomicroscopy. Nature 2004 ; 432 : 573-9.

7. Edeling MA, Smith C, Owen D. Life of a clathrin coat: insights from clathrin and AP structures. Nat Rev Mol Cell Biol $2006 ; 7$ : 32-44.

8. Marks B, Stowell MH, Vallis Y, et al. GTPase activity of dynamin and resulting conformation change are essential for endocytosis. Nature 2001 ; 410 : 231-5.

9. Braell WA, Schlossman DM, Schmid SL, Rothman JE. Dissociation of clathrin coats coupled to the hydrolysis of ATP: role of an uncoating ATPase. J Cell Biol 1984 ; 99 : 734-41.

10. Greene $L \varepsilon$, Eisenberg $\varepsilon$. Dissociation of clathrin from coated vesicles by the uncoating ATPase. J Biol Chem $1990 ; 265: 6682-7$.

11. Massol RH, Boll W, Griffin AM, Kirchhausen T. A burst of auxilin recruitment determines the onset of clathrincoated vesicle uncoating. Proc Natl Acad Sci USA $2006 ; 103: 10265-70$.

12. Rappoport JZ, Simon SM, Benmerah A. Understanding living clathrin-coated pits. Traffic 2004 ; 5 : 327-37.

13. Merrifield CJ. Seeing is believing: imaging actin dynamics at single sites of endocytosis. Trends Cell Biol $2004 ; 14$ : 352-8.

14. Kirchhausen T. Imaging endocytic clathrin structures in living cells. Trends Cell Biol 2009 ; 19 : 596-605.

15. Blondeau F, Ritter B, Allaire PD, et al. Tandem MS analysis of brain clathrin-coated vesicles reveals their critical involvement in synaptic vesicle recycling. Proc Natl Acad Sci USA $2004 ; 101$ : 3833-8.

16. Borner GH, Harbour M, Hester S, et al. Comparative proteomics of clathrin-coated vesicles. J Cell Biol $2006 ; 175: 571-8$

17. Huang F, Khvorova A, Marshall W, Sorkin A. Analysis of clathrin-mediated endocytosis of epidermal growth factor receptor by RNA interference. J Biol Chem 2004 ; 279 : 16657-61.
18. Mettlen M, Stoeber M, Loerke D, et al. Endocytic accessory proteins are functionally distinguished by their differential effects on the maturation of clathrin-coated pits. Mol Biol Cell 2009; $20: 3251-60$.

19. Ehrlich M, Boll W, Van Oijen A, et al. Endocytosis by random initiation and stabilization of clathrin-coated pits. Cell $2004 ; 118: 591-605$.

20. Loerke D, Mettlen M, Yarar D, et al. Cargo and dynamin regulate clathrin-coated pit maturation. PLoS Biol 2009 ; $7:$ e 57 .

21. Motley A, Bright NA, Seaman MN, Robinson MS. Clathrinmediated endocytosis in AP-2-depleted cells. J Cell Biol 2003 ; 162 : 909-18.

22. Boucrot $\varepsilon$, Saffarian $S$, Zhang R, Kirchhausen T. Roles of AP-2 in clathrin-mediated endocytosis. PLoS One 2010 ; $5:$ el0597.

23. Heuser J. Three-dimensional visualization of coated vesicle formation in fibroblasts. J Cell Biol $1980 ; 84: 560-83$.

24. Ford MG, Pearse BM, Higgins MK, et al. Simultaneous binding of Ptdlns (4,5)P2 and clathrin by AP180 in the nucleation of clathrin lattices on membranes. Science $2001 ; 291$ : 1051-5.

25. Ford MG, Mills IG, Peter BJ, et al. Curvature of clathrincoated pits driven by epsin. Nature 2002 ; 419 : 361-6.

26. Peter BJ, Kent HM, Mills IG, et al. BAR domains as sensors of membrane curvature: the amphiphysin BAR structure. Science $2004 ; 303: 495-9$.

27. Shimada A, Niwa H, Tsujita K, et al. Curved EFC/F-BARdomain dimers are joined end to end into a filament for membrane invagination in endocytosis. Cell 2007 ; $129: 761-72$.

28. Henne WM, Boucrot $\varepsilon$, Meinecke M, et al. FCHo proteins are nucleators of clathrin-mediated endocytosis. Science 2010 ; 328: 1281-4.

29. Reider A, Barker SL, Mishra SK, et al. Sypl is a conserved endocytic adaptor that contains domains involved in cargo selection and membrane tubulation. EMBO J 2009; $28: 3103-16$.

30. Stimpson HE, Toret CP, Cheng AT, et al. Early-arriving Syplp and Edelp function in endocytic site placement and formation in budding yeast. Mol Biol Cell $2009 ; 20: 4640-51$.

31. Frost A, Unger VM, De Camilli P. The BAR domain superfamily: membrane-molding macromolecules. Cell 2009; 137 : 191-6.

32. Henne WM, Kent HM, Ford MG, et al. Structure and analysis of FCHo2 F-BAR domain: a dimerizing and membrane recruitment module that effects membrane curvature. Structure $2007 ; 15: 839-52$.

33. Benmerah A, Lamaze C. Endocytose : chaque voie compte ! Med Sci (Paris) $2002 ; 18: 1126-36$ 